\title{
Weakly directed self-avoiding walks
}

\author{
Axel Bacher ${ }^{1}$ and Mireille Bousquet-Mélou ${ }^{2}$ \\ ${ }^{1}$ LaBRI, Université Bordeaux 1, 351 cours de la Libération, 33405 Talence, France \\ ${ }^{2}$ CNRS, LaBRI, Université Bordeaux 1, 351 cours de la Libération, 33405 Talence, France
}

\begin{abstract}
We define a new family of self-avoiding walks (SAW) on the square lattice, called weakly directed walks. These walks have a simple characterization in terms of the irreducible bridges that compose them. We determine their generating function. This series has a complex singularity structure and in particular, is not D-finite. The growth constant is approximately 2.54 and is thus larger than that of all natural families of SAW enumerated so far (but smaller than that of general SAW, which is about 2.64). We also prove that the end-to-end distance of weakly directed walks grows linearly. Finally, we study a diagonal variant of this model.

Résumé. Nous définissons une nouvelle famille de chemins auto-évitants (CAE) sur le réseau carré, appelés chemins faiblement dirigés. Ces chemins ont une caractérisation simple en termes des ponts irréductibles qui les composent. Nous déterminons leur série génératrice. Cette série a une structure de singularités complexe et n'est en particulier pas D-finie. La constante de croissance est environ 2,54, ce qui est supérieur à toutes les familles naturelles de SAW étudiées jusqu'à présent, mais inférieur aux CAE généraux (dont la constante est environ 2,64). Nous prouvons également que la distance moyenne entre les extrémités du chemin croît linéairement. Enfin, nous étudions une variante diagonale du modèle.
\end{abstract}

Keywords: Enumeration - Self-avoiding walks

\section{Introduction}

A lattice walk is self-avoiding if it never visits twice the same vertex (Fig. 1). Self-avoiding walks (SAW) have attracted interest for decades, first in statistical physics, where they are considered as polymer models, and then in combinatorics and in probability theory [20]. However, their properties remain poorly understood in low dimension, despite the existence of remarkable conjectures. See [20] for dimension 5 and above.

On two-dimensional lattices, it is strongly believed that the number $c_{n}$ of $n$-step SAW and the average end-to-end distance $D_{n}$ of these walks satisfy

$$
c_{n} \sim \alpha \mu^{n} n^{\gamma} \quad \text { and } \quad D_{n} \sim \kappa n^{\nu}
$$

where $\gamma=11 / 32$ and $\nu=3 / 4$. Several independent, but so far not completely rigorous methods predict these values, like numerical studies [12, 24], comparisons with other models [6, 21], probabilistic arguments involving SLE processes [19], enumeration of SAW on random planar lattices [10]... The growth constant (or connective constant) $\mu$ is lattice-dependent, and believed to be $\sqrt{2+\sqrt{2}}$ for the honeycomb lattice, and another bi-quadratic number (approximately 2.64) for the square lattice [16]. 

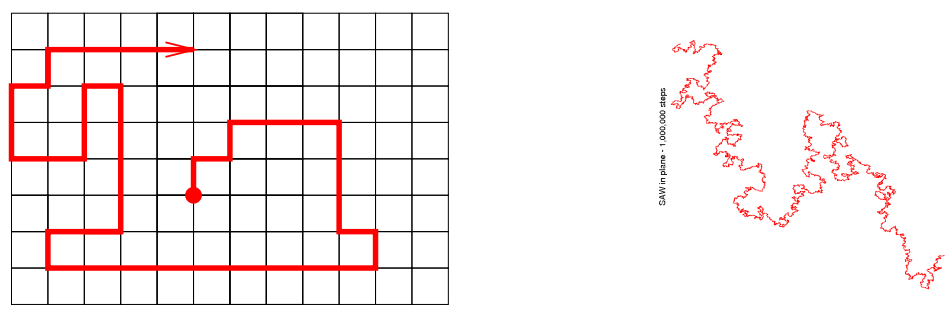

Fig. 1: A self-avoiding walk on the square lattice, and a (quasi-)random SAW of length 1,000,000, constructed by Kennedy using a pivot algorithm [17].

Given the difficulty of the problem, the study of restricted classes of SAW is natural, and probably as old as the interest in SAW itself. The rule of this game is to design new classes of SAW that have both:

- a natural description (to be conceptually pleasant),

- some structure (so that the walks can be counted, and their asymptotic properties determined).

The two simplest classes of SAW on the square lattice probably consist of directed and partially directed walks: a walk is directed if it involves at most two types of steps (for instance North and East), and partially directed if it involves at most three types of steps. (Partially directed walks play a prominent role in the definition of our weakly directed walks.) Among other solved classes, let us cite spiral SAW [22, 13] and prudent walks [3, 8, 7]. Each time such a new class is solved, one compares its properties to (1): have we reached with this class a large growth constant? Is the end-to-end distance of the walks sub-linear?

At the moment, the largest growth constant (about 2.48) is obtained with prudent SAW. However, this is beaten by certain classes whose description involves a (small) integer $k$, like SAW confined to a strip of height $k$ [1, 26], or SAW consisting of irreducible bridges of length at most $k$ [15, 18]. The structure of these walks is rather poor, which makes them little attractive from a combinatorial viewpoint. In the former case, they are described by a transfer matrix (the size of which increases exponentially with the height of the strip); in the latter case, the structure is even simpler, since these walks are just arbitrary sequences of irreducible bridges of bounded length. In both cases, improvements on the growth constant much rely on progresses in the computer power. Regarding asymptotic properties, almost all solved classes of SAW exhibit a linear end-to-end distance, with the exception of spiral walks. But there are very few such walks [13], as their growth constant is 1 .

With the weakly directed walks of this paper, we reach a growth constant of about 2.54. These walks are defined in the next section. Their generating function is given in Section 5, after some preliminary results on partially directed bridges (Sections 3 and 4). This series turns out to be much more complicated that the generating functions of directed and partially directed walks, which are rational: we prove that it has a natural boundary in the complex plane, and in particular is not D-finite (that is, it does not satisfy any linear differential equation with polynomial coefficients). However, we are able to derive from this series certain asymptotic properties of weakly directed walks, like their growth constant and average end-to-end distance (which we find, unfortunately, to grow linearly with the length). Finally, we perform in Section 6 a similar study for a diagonal variant of weakly directed walks.

Due to space constraints, most proofs are only sketched or even omitted in this abstract. Details will appear in the complete version of the paper. 


\section{Weakly directed walks: definition}

Let us denote by N, E, S and W the four square lattice steps. All walks in this paper are self-avoiding, so that this precision will often be omitted. For any subset $\mathcal{S}$ of $\{\mathrm{N}, \mathrm{E}, \mathrm{S}, \mathrm{W}\}$, we say that a (self-avoiding) walk is an $\mathcal{S}$-walk if all its steps lie in $\mathcal{S}$. We say that a SAW is directed if it involves at most two types of steps, and partially directed if it involves at most three types of steps. The definition of weakly directed walks stems for the following simple observations:

(i) between two visits to any given horizontal line, a NE-walk only takes $E$ steps,

(ii) between two visits to any given horizontal line, a NEW-walk only takes $E$ and $W$ steps.

Conversely, a walk satisfies (i) if and only if it is either a NE-walk or, symmetrically, a SE-walk. Similarly, a walk satisfies (ii) if and only if it is either a NEW-walk or, symmetrically, a SEW-walk. Conditions (i) and (ii) thus respectively characterize (up to symmetry) NE-walks and NEW-walks.

Definition 1 A walk is weakly directed if, between two visits to any given horizontal line, the walk is partially directed (that is, avoids at least one of the steps $\mathrm{N}, \mathrm{E}, \mathrm{S}, \mathrm{W}$ ).

Examples are shown in Fig.2
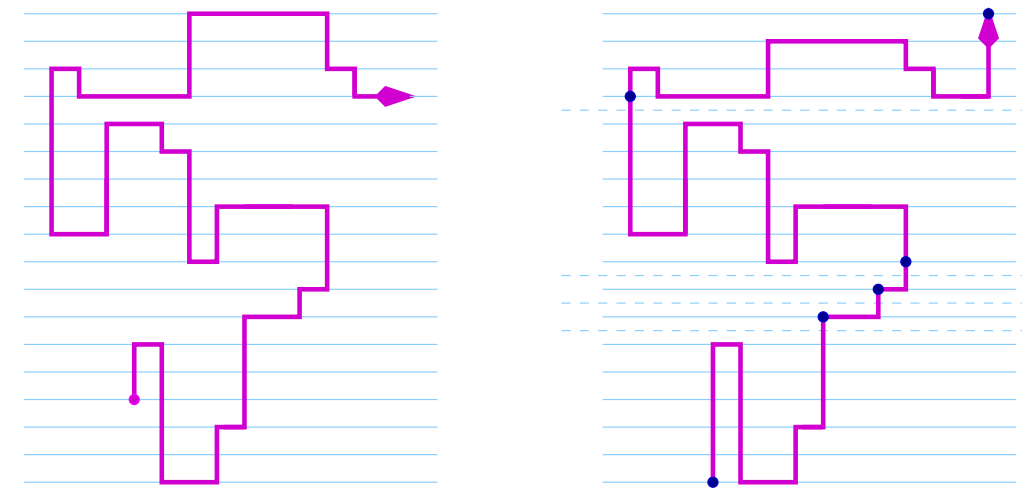

Fig. 2: Two weakly directed walks. The second one is a bridge, formed of 5 irreducible bridges. Observe that these irreducible bridges are partially directed.

We will primarily focus on the enumeration of weakly directed bridges. As we shall see, this does not affect the growth constant. A self-avoiding walk starting at $v_{0}$ and ending at $v_{n}$ is a bridge if all its vertices $v \neq v_{n}$ satisfy $h\left(v_{0}\right) \leq h(v)<h\left(v_{n}\right)$, where $h(v)$, the height of $v$, is its ordinate. Concatenating two bridges always gives a bridge. Conversely, every bridge can be uniquely factored into a sequence of irreducible bridges (bridges that cannot be written as the product of two non-empty bridges). This factorization is obtained by cutting the walk above each horizontal line of height $n+1 / 2$ (with $n \in \mathbb{Z}$ ) that the walk intersects only once (Fig. 2, right). It is known that the growth constant of bridges is the same as for general self-avoiding walks [20]. Generally speaking, the fact that bridges can be freely concatenated makes them useful objects in the study of self-avoiding walks [14, 15, 18, 19, 20].

The following result shows that the enumeration of weakly directed bridges boils down to the enumeration of (irreducible) partially directed bridges. 
Proposition 2 A bridge is weakly directed if and only if each of its irreducible bridges is partially directed (that is, avoids at least one of the steps $\mathrm{N}, \mathrm{E}, \mathrm{S}, \mathrm{W}$ ).

We discuss in Section 6 a variant of weakly directed walks, where we constrain the walk to be partially directed between two visits to the same diagonal line (Fig. 3). The notion of bridges is adapted accordingly, by defining the height of a vertex as the sum of its coordinates. We will refer to this model as the diagonal model, and to the original one as the horizontal model. There is, however, no simple counterpart of Proposition 2 : a (diagonal) bridge whose irreducible bridges are partially directed is always weakly directed, but the converse is not true, as can be seen in Fig. 3 . Thus bridges with partially directed irreducible bridges form a proper subclass of weakly directed bridges. We will enumerate this subclass, and study its asymptotic properties.

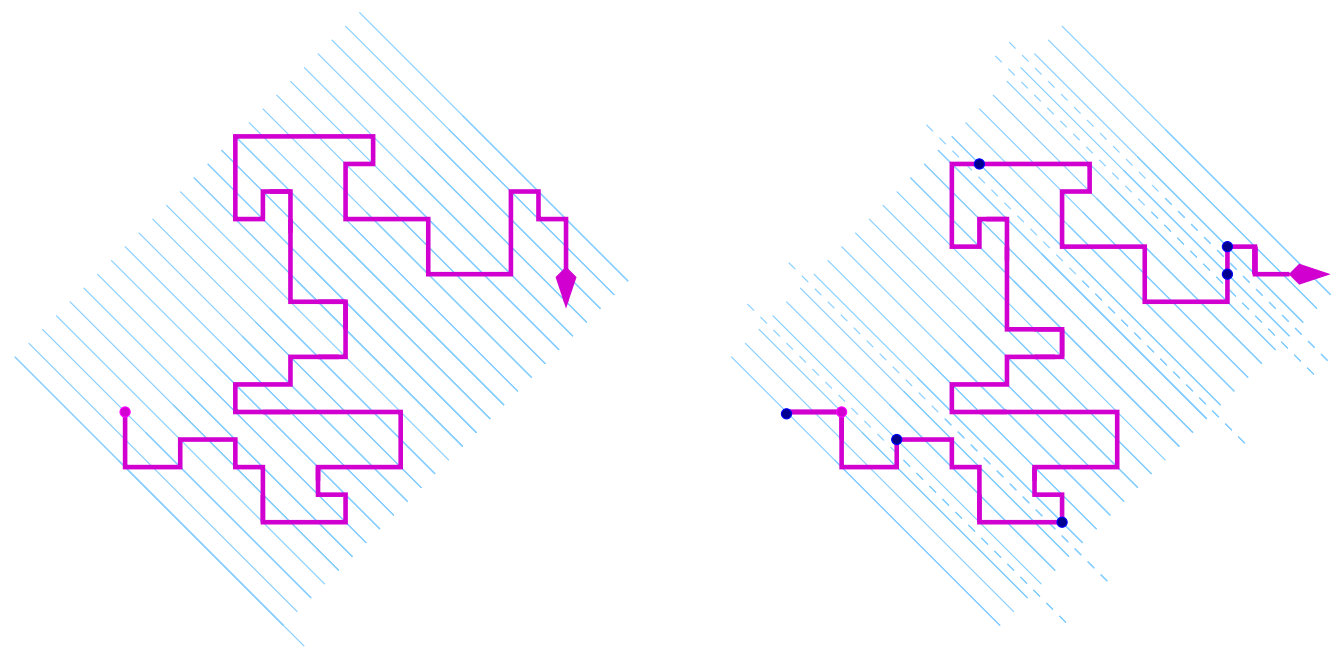

Fig. 3: Two weakly directed walks in the diagonal model. The second one is a bridge, factored into 6 irreducible bridges. Observe that the third irreducible bridge is not partially directed.

\section{Partially directed bridges: a step-by-step approach}

Let us equip the square lattice $\mathbb{Z}^{2}$ with its standard coordinate system. With each model (horizontal or diagonal) is associated a notion of height: the height of a vertex $v$, denoted $h(v)$, is its ordinate in the horizontal model, while in the diagonal model, it is the sum of its coordinates. Recall that a walk, starting at $v_{0}$ and ending at $v_{n}$, is a bridge if all its vertices $v \neq v_{n}$ satisfy $h\left(v_{0}\right) \leq h(v)<h\left(v_{n}\right)$. If the weaker inequality $h\left(v_{0}\right) \leq h(v) \leq h\left(v_{n}\right)$ holds for all $v$, we say the walk is a pseudo-bridge. Note that nonempty bridges are obtained by adding a step of height 1 to a pseudo-bridge (a $\mathrm{N}$ step in the horizontal model, a $\mathrm{N}$ or $\mathrm{E}$ step in the diagonal model). It is thus equivalent to count bridges or pseudo-bridges.

By Proposition 2 the enumeration of weakly directed bridges boils down to the enumeration of (irreducible) partially directed bridges. In this section and the following one, we address the enumeration of these building blocks, first in a rather systematic way based on a step-by-step construction, then in a more combinatorial way based on heaps of cycles. 

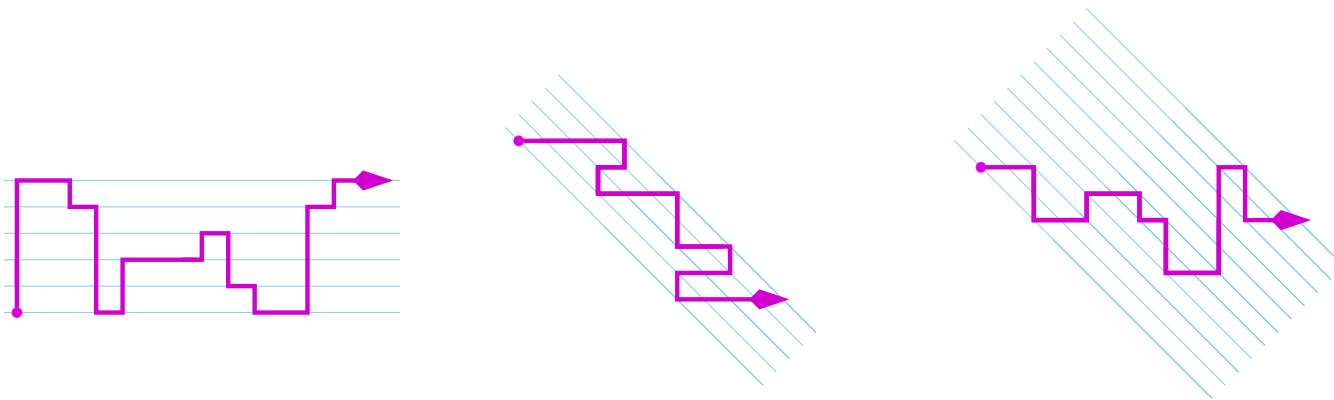

Fig. 4: A NES-pseudo-bridge in the horizontal model. (b) An ESW-pseudo-bridge in the diagonal model. (c) A NES-pseudo-bridge in the diagonal model.

As partially directed walks are defined by the avoidance of (at least) one step, there are four kinds of these. Hence, in principle, we should count, for each model (horizontal and diagonal), four families of partially directed bridges. However, in the horizontal model, there exists no ESW-bridge, and every NEW-walk is a pseudo-bridge. The latter class of walks is very easy to count. Moreover, a symmetry transforms NES-bridges into NSW-bridges, so that there is really one class of bridges that we need to count. In the diagonal model, we need to count ESW-bridges (which are equivalent to NSW-bridges by a diagonal symmetry) and NES-bridges (which are equivalent to NEW-bridges). Finally, to avoid certain ambiguities, we need to count ES-bridges, but this has already been done in [5].

From now on, the starting point of our walks is always at height 0 . The height of a walk is then defined to be the maximal height reached by its vertices.

\subsection{NES-bridges in the horizontal model}

Proposition 3 Let $k \geq 0$. In the horizontal model, the length generating function of NES-pseudo-bridges of height $k$ is

$$
B^{(k)}(t)=\frac{t^{k}}{G_{k}(t)}
$$

where $G_{k}(t)$ is the sequence of polynomials defined by

$$
G_{-1}=1, \quad G_{0}=1-t, \quad \text { and for } k \geq 0, \quad G_{k+1}=\left(1-t+t^{2}+t^{3}\right) G_{k}-t^{2} G_{k-1} .
$$

Equivalently,

$$
\sum_{k \geq 0} \frac{v^{k} t^{k}}{B^{(k)}(t)}=\sum_{k \geq 0} v^{k} G_{k}=\frac{1-t-t^{2} v}{1-\left(1-t+t^{2}+t^{3}\right) v+t^{2} v^{2}}
$$

or

$$
B^{(k)}(t)=\frac{U-\bar{U}}{((1-t) U-t) U^{k}-((1-t) \bar{U}-t) \bar{U}^{k}},
$$

where

$$
U=\frac{1-t+t^{2}+t^{3}-\sqrt{\left(1-t^{4}\right)\left(1-2 t-t^{2}\right)}}{2 t}
$$

is a root of $t u^{2}-\left(1-t+t^{2}+t^{3}\right) u+t=0$ and $\bar{U}:=1 / U$ is the other root of this polynomial. 
Proof: Let $\mathcal{T}$ be the set of NES-walks that end with an E step, and in which each vertex $v$ satisfies $0 \leq h(v) \leq k$. Let $\mathcal{T}_{i}$ be the subset of $\mathcal{T}$ consisting of walks that end at height $i$. Let $T_{i}(t) \equiv T_{i}$ be the length generating function of $\mathcal{T}_{i}$, and define the bivariate generating function

$$
T(t ; u) \equiv T(u)=\sum_{i=0}^{k} T_{i}(t) u^{i} .
$$

This series counts walks of $\mathcal{T}$ by their length and the height of their endpoint. Pseudo-bridges walks of height $k$ containing at least one $\mathrm{E}$ step are obtained by adding a sequence of $\mathrm{N}$ steps of appropriate length to a walk of $\mathcal{T}$, and this gives

$$
B^{(k)}(t)=t^{k}+\sum_{i=0}^{k} T_{i}(t) t^{k-i}=t^{k}(1+T(1 / t))
$$

(the term $t^{k}$ accounts for the walk formed of $k$ consecutive $\mathrm{N}$ steps.)

A step-by-step construction of the walks of $\mathcal{T}$ yields the following lemma.

Lemma 4 Let $\bar{u}=1 / u$. The series $T(t ; u)$, denoted $T(u)$ for short, satisfies the following equation:

$$
\left(1-\frac{u t^{2}}{1-t u}-\frac{t}{1-t \bar{u}}\right) T(u)=t \frac{1-(t u)^{k+1}}{1-t u}-t \frac{(t u)^{k+1}}{1-t u} T(1 / t)-\frac{t^{2} \bar{u}}{1-t \bar{u}} T(t) .
$$

The equation of Lemma 4 is easily solved using the kernel method (see e.g. [2, 4, 23]). The kernel of the equation is the coefficient of $T(u)$, namely

$$
1-\frac{u t^{2}}{1-t u}-\frac{t}{1-t \bar{u}}
$$

It vanishes when $u=U$ and $u=\bar{U}:=1 / U$, where $U$ is defined in the lemma. Since $T(u)$ is a polynomial in $u$, the series $T(U)$ and $T(\bar{U})$ are well-defined. Replacing $u$ by $U$ or $\bar{U}$ in the functional equation cancels the left-hand side, and hence the right-hand side. One thus obtains two linear equations between $T(t)$ and $T(1 / t)$, which involve the series $U$. Solving them gives in particular the value of $T(1 / t)$, and thus of $B^{(k)}(t)$ (thanks to (2)). This provides the second expression of $B^{(k)}(t)$ given in the lemma. The other results easily follow, using elementary arguments about linear recurrence relations and rational generating functions.

\subsection{ESW-bridges in the diagonal model}

Proposition 5 Let $k \geq 0$. In the diagonal model, the length generating function of ESW-pseudo-bridges of height $k$ is

$$
B_{1}^{(k)}(t)=\frac{t^{k}}{G_{k}(t)}
$$

where $G_{k}(t)$ is the sequence of polynomials defined by

$$
G_{0}=1, \quad G_{1}=1-t^{2} \quad \text { and for } k \geq 1, \quad G_{k+1}=\left(1+t^{2}\right) G_{k}-t^{2}\left(2-t^{2}\right) G_{k-1} .
$$


The length generating function of NES-pseudo-bridges of height $k$ is

$$
B_{2}^{(k)}(t)=\left(2-t^{2}\right)^{k} B_{1}^{(k)}(t)
$$

Finally, the length generating function of ES-pseudo-bridges of height $k$ is

$$
B_{0}^{(k)}(t)=\frac{t^{k}}{F_{k}(t)},
$$

where the sequence $F_{k}(t)$ is defined by $F_{-1}=1, F_{0}=1$, and $F_{k+1}=F_{k}-t^{2} F_{k-1}$ for $k \geq 0$.

\section{Partially directed bridges via heaps of cycles}

In this section, we give alternative (and more combinatorial) proofs of the results of Section 3 . In particular, these proofs explain why the numerators of series counting partially directed bridges of height $k$ are so simple $\left(t^{k}\right.$ or $t^{k}\left(2-t^{2}\right)^{k}$, depending on the model).

Let $\Gamma=(V, E)$ be a directed graph. To each edge of this graph, we associate a weight taken in some commutative ring (typically, a ring of formal power series). A cycle of $\Gamma$ is a path ending at its starting point, taken up to a cyclic permutation. A path is self-avoiding if it does not visit the same vertex twice. A self-avoiding cycle is called an elementary cycle. Two paths are disjoint if their vertex sets are disjoint. The weight $w(\pi)$ of a path (or cycle) $\pi$ is the product of the weights of its edges. A configuration of cycles $\gamma=\left\{\gamma_{1}, \ldots, \gamma_{r}\right\}$ is a set of pairwise disjoint elementary cycles. The signed weight of $\gamma$ is

$$
\widetilde{w}(\gamma):=(-1)^{r} \prod_{i=1}^{r} w\left(\gamma_{i}\right)
$$

For two vertices $i$ and $j$, denote by $W_{i, j}$ the generating function of paths going from from $i$ to $j$ :

$$
W_{i, j}=\sum_{\pi: i \sim j} w(\pi) .
$$

We assume that this sum is well-defined, which is always the case when considering length generating functions.

Proposition 6 The generating function of paths going from $i$ to $j$ in the weighted digraph $\Gamma$ is

$$
W_{i, j}=\frac{N_{i, j}}{G}
$$

where $G=\sum_{\gamma} \widetilde{w}(\gamma)$ is the signed generating function of configuration of cycles, and

$$
N_{i, j}=\sum_{\eta, \gamma} w(\eta) \widetilde{w}(\gamma),
$$

where $\eta$ is a self-avoiding path going from $i$ to $j$ and $\gamma$ is a configuration of cycles disjoint from $\eta$. 
This result can be proved as follows: one first identifies $N_{i, j}$ as the $(i, j)$ coefficient of the matrix $(1-$ $A)^{-1}$, where $A$ is the adjacency matrix of $\Gamma$. Thanks to standard linear algebra, this coefficient can be expressed in terms of the determinant of $(1-A)$ and one of its cofactors. A simple expansion of these as sums over permutations shows that the determinant is $G$, and the cofactor $N_{i, j}$. Proposition 6 can also be proved without any reference to linear algebra, using the theory of partially commutative monoids, or heaps of pieces [11, 25]. In this context, configurations of cycles are called trivial heaps of cycles. This justifies the title of this section.

\subsection{Bridges with large down steps}

Let $\Gamma_{k}$ be the graph with vertices $\{0, \ldots, k\}$ and with the following weighted edges:

- ascending edges of height $1, i \rightarrow i+1$, with weight $A$, for $i=0, \ldots, k-1$;

- descending edges of height $h, i \rightarrow i-h$, with weight $D_{h}$, for $i=h, \ldots, k$ and $h \geq 0$.

For $k \geq 0$, denote by $C^{(k)}$ the generating function of paths from 0 to $k$ in the graph $\Gamma_{k}$. These paths may be seen as pseudo-bridges of height $k$ with general down steps.

Lemma 7 The generating function of pseudo-bridges of height $k$ is

$$
C^{(k)}=\frac{A^{k}}{H_{k}}
$$

where the generating function of the denominators $H_{k}$ is

$$
\sum_{k \geq 0} H_{k} v^{k}=\frac{1-D(v A)}{1-v+v D(v A)}
$$

with $D(v)$ the generating function of descending steps:

$$
D(v)=\sum_{h \geq 0} D_{h} v^{h}
$$

Proof: With the notation of Proposition 6 the series $C^{(k)}$ reads $N_{0, k} / G$. Since all ascending edges have height 1 , the only self-avoiding path from 0 to $k$ consists of $k$ ascending edges, and has weight $A^{k}$. As it visits every vertex of the graph, the only configuration of cycles disjoint from it is the empty configuration. Therefore, the numerator $N_{0, k}$ is simply $A^{k}$. The elementary cycles consist of a descending step of height, say, $h$, followed by $h$ ascending steps. The weight of this cycle is $D_{h} A^{h}$.

To underline the dependance of our graph in $k$, denote by $H_{k}$ the denominator $G$ of Proposition 6 Consider a configuration of cycles of $\Gamma_{k}$ : either the vertex $k$ is free, or it is occupied by a cycle; this gives the following recurrence relation, valid for $k \geq 0$ :

$$
H_{k}=H_{k-1}-\sum_{h=0}^{k} D_{h} A^{h} H_{k-h-1} .
$$

with the initial condition $H_{-1}=1$. This is equivalent to 3 . 


\subsection{Partially directed self-avoiding walks as arbitrary paths}

It is not straightforward to apply Proposition 6 (or Lemma 7) to the enumeration of partially directed bridges, because of the self-avoidance condition. To circumvent this difficulty, we will first prove that partially directed self-avoiding walks are arbitrary paths on a graph with generalized steps. We only deal with the horizontal model, but the diagonal model can be addressed in a similar way.

Let us say that a NES-walk is proper if it neither begins nor ends with a S step. All NES-pseudobridges are proper, whether in the horizontal or diagonal model. The following lemma explains how to see proper NES-walks as sequences of generalized steps.

Lemma 8 Every proper NES-walk has a unique factorization into $\mathrm{N}$ steps and nonempty proper $\mathrm{ES}$ walks with no consecutive $\mathrm{E}$ steps.

This result, combined with Lemma 7 gives an alternative proof of Proposition 3

\section{Weakly directed walks: the horizontal model}

We now return to the weakly directed walks defined in Section 2 . We determine their generating function, study their asymptotic number and average end-to-end distance, and finally prove that the generating function we have obtained has infinitely many singularities, and hence, cannot be D-finite.

\subsection{Generating function}

By combining Propositions 2 and 3 it is now easy to count weakly directed bridges.

Proposition 9 In the horizontal model, the generating function of weakly directed bridges is:

$$
W(t)=\frac{1}{1+t-\frac{2 t B}{1+t B}}
$$

where $B:=\sum_{k \geq 0} B^{(k)}(t)$ is the generating function of NES-pseudo-bridges, given by Proposition 3

The generating function of general weakly directed walks is a bit more involved, but the numbers of weakly directed walks and bridges of length $n$ only differ asymptotically by a multiplicative constant.

\subsection{Asymptotic results}

Proposition 10 The generating function $W$ of weakly directed bridges, given in Proposition 9 is meromorphic in the disk $\mathcal{D}=\{z:|z|<\sqrt{2}-1\}$. It has a unique dominant pole in this disk, $\rho \simeq 0.3929$. This pole is simple. Consequently, the number $w_{n}$ of weakly directed bridges of length $n$ satisfies $w_{n} \sim \kappa \mu^{n}$, with $\mu=1 / \rho \simeq 2.5447$.

Let $N_{n}$ denote the number of irreducible bridges in a random weakly directed bridge of length $n$. The mean and variance of $N_{n}$ satisfy:

$$
\mathbb{E}\left(N_{n}\right) \sim \mathfrak{m} n, \quad \mathbb{V}\left(N_{n}\right) \sim \mathfrak{s}^{2} n,
$$

where $\mathfrak{m} \simeq 0.318$ and $\mathfrak{s}^{2} \simeq 0.7$, and the random variable $\frac{N_{n}-\mathfrak{m} n}{\mathfrak{s} \sqrt{n}}$ converges in law to a standard normal distribution. In particular, the average end-to-end distance, being bounded from below by $\mathbb{E}\left(N_{n}\right)$, grows linearly with $n$. 
We have designed an algorithm for the random generation of weakly directed bridges, using a Boltzmann sampler [9]. A sample output of this algorithm is shown in Figure 5 . confirming the linear form of weakly directed bridges.

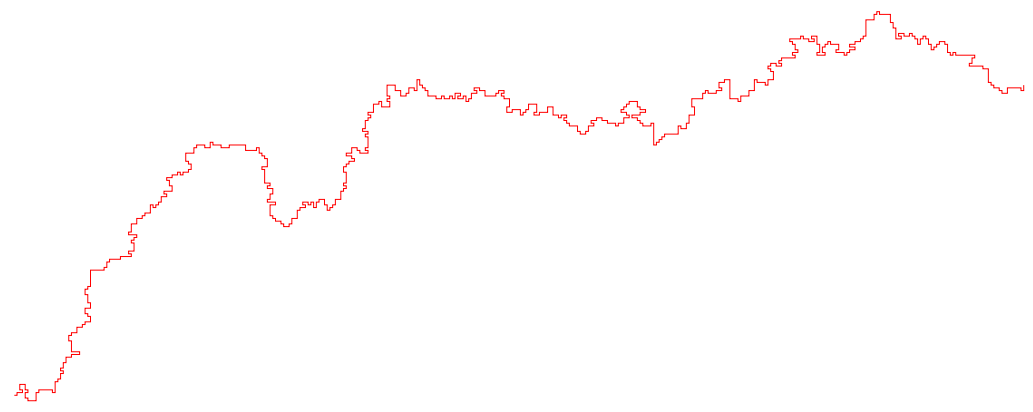

Fig. 5: A random weakly directed bridge of length 1009 , rotated by $90^{\circ}$.

\subsection{Nature of the series}

Proposition 11 The generating function B of NES-pseudo-bridges, given in Proposition 3 converges around 0 and has a meromorphic continuation in $\mathbb{C} \backslash \mathcal{E}$, where $\mathcal{E}$ consists of the two real intervals $[-\sqrt{2}-$ $1,-1]$ and $[\sqrt{2}-1,1]$, and of the curve

$$
\mathcal{E}_{0}=\left\{x+i y: x \geq 0, y^{2}=\frac{1-x^{2}-2 x^{3}}{1+2 x}\right\} .
$$

This curve, shown in Fig. 6, is a natural boundary of B. That is, every point of $\mathcal{E}_{0}$ is a singularity of $B$.

The above statements hold as well for the generating function $W$ of weakly directed bridges. In particular, neither $B$ nor $W$ is D-finite.

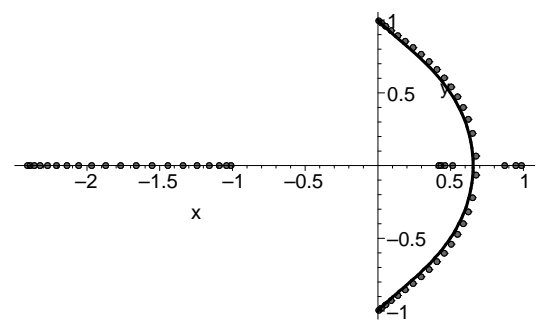

Fig. 6: The curve $\mathcal{E}_{0}$ and the zeroes of the polynomial $G_{20}$.

\section{The diagonal model}

We have defined weakly directed walks in Section 2 by requiring that the portion of the walk joining two visits to the same diagonal is partially directed. Here, we study a proper subclass of these walks, consisting of bridges formed of partially directed irreducible bridges. 
Proposition 12 The generating function of bridges formed of partially directed irreducible bridges is

$$
W_{\Delta}(t)=\frac{1}{1+2 t-\frac{2 t B_{1}}{1+t B_{1}}-\frac{4 t B_{2}}{1+2 t B_{2}}+\frac{2 t B_{0}}{1+t B_{0}}},
$$

where the series $B_{i}=\sum_{k \geq 0} B_{i}^{(k)}(t)$ are given in Proposition 5

The growth constant is found to be a bit smaller than in the horizontal model (about 2.5378). The end-to-end distance is again linear.

\section{References}

[1] S. E. Alm and S. Janson. Random self-avoiding walks on one-dimensional lattices. Comm. Statist. Stochastic Models, 6(2):169-212, 1990.

[2] C. Banderier, M. Bousquet-Mélou, A. Denise, P. Flajolet, D. Gardy, and D. Gouyou-Beauchamps. Generating functions for generating trees. Discrete Math., 246(1-3):29-55, 2002.

[3] M. Bousquet-Mélou. Families of prudent self-avoiding walks. J. Combin. Theory Ser. A, 117(3):313344, 2010. Arxiv:0804.4843.

[4] M. Bousquet-Mélou and M. Petkovšek. Linear recurrences with constant coefficients: the multivariate case. Discrete Math., 225(1-3):51-75, 2000.

[5] M. Bousquet-Mélou and Y. Ponty. Culminating paths. Discrete Math. Theoret. Comput. Sci., 10(2), 2008. ArXiv:0706.0694.

[6] P. G. de Gennes. Exponents for the excluded volume problem as derived by the Wilson method. Phys. Lett. A, 38(5):339-340, 1972.

[7] J. C. Dethridge and A. J. Guttmann. Prudent self-avoiding walks. Entropy, 8:283-294, 2008.

[8] E. Duchi. On some classes of prudent walks. In FPSAC'05, Taormina, Italy, 2005.

[9] Ph. Duchon, Ph. Flajolet, G. Louchard, and G. Schaeffer. Boltzmann samplers for the random generation of combinatorial structures. Combin. Probab. Comput., 13(4-5):577-625, 2004.

[10] B. Duplantier and I. K. Kostov. Geometrical critical phenomena on a random surface of arbitrary genus. Nucl. Phys. B, 340(2-3):491-541, 1990.

[11] D. Foata. A noncommutative version of the matrix inversion formula. Adv. Math., 31:330-349, 1979.

[12] A. J. Guttmann and A. R. Conway. Square lattice self-avoiding walks and polygons. Ann. Comb., 5(3-4):319-345, 2001.

[13] A. J. Guttmann and N. C. Wormald. On the number of spiral self-avoiding walks. J. Phys. A: Math. Gen., 17:L271-L274, 1984. 
[14] J. M. Hammersley and D. J. A. Welsh. Further results on the rate of convergence to the connective constant of the hypercubical lattice. Q. J. Math., Oxf. II. Ser., 13:108-110, 1962.

[15] I. Jensen. Improved lower bounds on the connective constants for two-dimensional self-avoiding walks. J. Phys. A: Math. Gen., 37(48):11521-11529, 2004.

[16] I. Jensen and A. J. Guttmann. Self-avoiding polygons on the square lattice. J. Phys. A, 32(26):48674876, 1999.

[17] T. Kennedy. A faster implementation of the pivot algorithm for self-avoiding walks. J. Statist. Phys., 106(3-4):407-429, 2002.

[18] H. Kesten. On the number of self-avoiding walks. J. Math. Phys., 4(7):960-969, 1963.

[19] G. F. Lawler, O. Schramm, and W. Werner. On the scaling limit of planar self-avoiding walk. In Fractal geometry and applications: a jubilee of Benoît Mandelbrot, Part 2, volume 72 of Proc. Sympos. Pure Math., pages 339-364. Amer. Math. Soc., Providence, RI, 2004.

[20] N. Madras and G. Slade. The self-avoiding walk. Probability and its Applications. Birkhäuser Boston Inc., Boston, MA, 1993.

[21] B. Nienhuis. Exact critical point and critical exponents of $o(n)$ models in two dimensions. Phys. Rev. Lett., 49(15):1062-1065, 1982.

[22] V. Privman. Spiral self-avoiding walks. J. Phys. A: Math. Gen., 16(15):L571-L573, 1983.

[23] H. Prodinger. The kernel method: a collection of examples. Sém. Lothar. Combin., 50:Art. B50f, 19 pp. (electronic), 2003/04.

[24] A. Rechnitzer and E. J. Janse van Rensburg. Canonical Monte Carlo determination of the connective constant of self-avoiding walks. J. Phys. A, Math. Gen., 35(42):L605-L612, 2002.

[25] X. G. Viennot. Heaps of pieces, I : Basic definitions and combinatorial lemmas, volume 1234/1986, pages 321-350. Springer Berlin / Heidelberg, 1986.

[26] D. Zeilberger. Symbol-crunching with the transfer-matrix method in order to count skinny physical creatures. Integers, pages A9, 34pp. (electronic), 2000. 